zkm Praxis Integrative Onkologie

\title{
Das Essener Modell der Integrativen Onkologie
}

Integrative Krebstherapie - Evidenzbasiert und supportiv zur konventionellen Krebstherapie für mehr Lebensqualität und Patientenzufriedenheit Petra Voiß, Kyung-Eun Choi, Anna Paul, Gustav Dobos 
„Was wollen Sie denn mit Naturheilkunde? Sie sind ernsthaft krank!“ - „Am besten Sie ändern gar nichts an Ihrem Leben, das bringt uns nur unsere Therapie durcheinander ..." - Solche und ähnliche Kommentare bekommen Krebspatienten nicht selten zu hören, wenn sie ihren Arzt fragen, was sie selbst in ihrer Situation für sich tun können. Dabei haben viele von ihnen den Wunsch, selbst etwas zu ihrer Gesundung beizutragen [1] - etwas Positives, denn sie wissen, dass der Kampf gegen eine Tumorerkrankung zunächst einmal ein Krieg ist - mit zerstörerischen Mitteln, die sich gegen den Feind im eigenen Körper richten.

$70 \%$ der deutschen Krebspatienten, so Zahlen der Deutschen Krebshilfe, suchen Hilfe bei alternativen oder komplementären Therapien - eine riskante Strategie, wenn sie von ärztlicher Seite aus nicht kompetent koordiniert und begleitet wird. Groß sind die Risiken unerwünschter Wechselwirkungen mit der onkologischen Therapie, die im schlimmsten Fall sogar unwirksam werden kann - z. B. durch hohe Vitamindosen, Grapefruitsaft oder grünen Tee, die von medizinischen Laien i.d.R. als durchweg gesund und ungefährlich eingeschätzt werden.

Doch leider sprechen die wenigsten Patienten aus Angst vor Ablehnung mit ihrem Onkologen über solche eigenmächtigen Behandlungsversuche. Und selbst wenn sie es täten, würden viele Krebsmediziner von begleitenden komplementärmedizinischen Methoden abraten, weil sie wenig Wissen über deren positives Potenzial haben.

Das ändert sich gerade. Evidenzbasierte Verfahren der Naturheilkunde und Komplementärmedizin finden immer häufiger ihren Weg in die Leitlinien der Krebsmedizin. Denn immer stärker wird deutlich, dass das nicht nur der Wunsch der meisten Patienten ist, sondern diese „integrative“ Form der Onkologie auch einen erheblichen Beitrag dazu leisten kann, belastende Nebenwirkungen zu lindern, die Lebensqualität zu verbessern und Risiken durch „unautorisierte“ Selbstbehandlungen zu vermeiden.

Die Wurzeln einer „Integrativen Onkologie“ liegen in den USA. Dort fordern Patienten seit den 1990er-Jahren verstärkt die Einbindung traditioneller Heilverfahren in ihre Behandlung ein. Alle renommierten Cancer Center haben deshalb mittlerweile Abteilungen für Integrative Onkologie etabliert, z.B. das Memorial SloanKettering Cancer Center in New York. Der kanadische Radiologe Stephen Sagar beschrieb die Integrative Onkologie als „science and a philosophy that focuses on the complexity of the health of cancer patients and proposes a multitude of approaches to accompany the conventional therapies of surgery, chemotherapy, molecular therapeutics, and radiotherapy to facilitate health. As such, integrative oncology involves thinking outside the box" [2].

Die erste Arbeitsgruppe für komplementäre Krebsmedizin hat die einflussreiche „American Urological Association“ bereits in den

\section{Die Integrative Onkologie ist immer}

\section{evidenzbasiert. Sie ersetzt in keinem}

\section{Fall die konventionelle onkologische}

Therapie, sondern ergänzt sie.

\begin{abstract}
Zusammenfassung
$70 \%$ der deutschen Krebspatienten suchen auch bei komplementären und alternativen Therapien Hilfe. Das setzt eine kompetente Koordination von ärztlicher Seite voraus, da bei der Anwendung Risiken wie unerwünschte Wechselwirkungen mit der onkologischen Therapie bestehen.

Die Integrative Onkologie hat als Ziele die Verbesserung der Lebensqualität und der Zufriedenheit der Patienten, die Primär- und Sekundärprophylaxe, ein verbessertes Nebenwirkungsmanagement, ggf. eine Verbesserung der Prognose, eine wissenschaftliche Evaluierung, wissenschaftlichen Austausch sowie die Ausbildung von Ärzten und Gesundheitsberufen.

Die Autoren stellen das Essener Modell der Integrativen Onkologie vor, das von gut $60 \%$ der Essener Patientinnen wahrgenommen wird. Die Mind-Body-Medizin bildet eine wichtige Basis der integrativen Behandlung.
\end{abstract}

1990er-Jahren ins Leben gerufen - da in den USA 80\% der Männer mit Prostatakrebs ergänzende Therapien nachfragten [3]. Im Jahr 2003 gründete sich dann die „International Society for Integrative Oncology (SIO)“ als internationale Fachgesellschaft, die seither entsprechende Forschungsansätze und Erfahrungen aus der klinischen Praxis koordiniert und diskutiert.

\section{Ziele der Integrativen Onkologie}

Die Ziele der Integrativen Onkologie sind die Verbesserung der Lebensqualität der Patienten, die Verbesserung der Patientenzufriedenheit, Primär- und Sekundärprophylaxe, verbessertes Nebenwirkungsmanagement, ggf. eine Verbesserung der Prognose, die wissenschaftliche Evaluierung, wissenschaftlicher Austausch auf nationaler und internationaler Ebene sowie die Ausbildung ärztlicher Kollegen und weiterer Gesundheitsberufe.

2007 wurden von der SIO auf dieser Basis die ersten Leitlinien für die klinische Praxis veröffentlicht, 2009 erschien ein Update [4-6]. 2013 wurden überarbeitete Leitlinien für die integrative Behandlung von Lungenkrebs publiziert, und 2014 erschien im Journal of the National Cancer Institute Monographs eine Sonderausgabe zum Thema Brustkrebs [7].

In den USA wird die komplementärmedizinische Forschung staatlich gefördert: Allein im Bereich Krebs geben das National Center for Complementary and Integrative Health $(\mathrm{NCClH})$ und das National Cancer Institute jährlich jeweils rund 125 Mio. Dollar dafür aus.

In Deutschland ist die Forschungsfinanzierung im Bereich Integrativer Medizin nach wie vor nur mithilfe von Stiftungen möglich. Dennoch wächst die Zahl relevanter Studien, die Basis einer in Arbeit befindlichen Leitlinie „Komplementärmedizin in der Behandlung von onkologischen PatientInnen“ unter dem Dach der Deutschen Krebsgesellschaft sind. Der Lehrstuhl für Naturheilkunde und Integrative Medizin an der Universität Duisburg-Essen hat in Zusammenarbeit mit der Deutschen Gesellschaft für Naturheilkunde und Unterstützung der Rut- und Klaus-Bahlsen-Stiftung zusätzlich eine Taskforce „Naturheilkunde und Komplementärmedizin in medizini- 


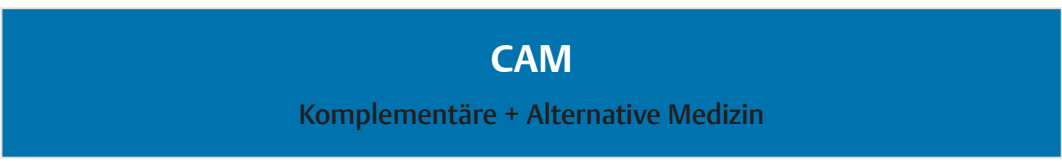

Komplementär

in Ergänzung zur wissenschaftlich begründeten Medizin
Alternativ

anstelle der wissenschaftlich begründeten Medizin

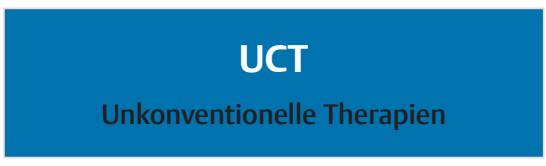

Unkonventionell

unbewiesene Außenseiter-Methoden

Komplementäre + Konventionelle Onkologie = Integrative Onkologie

Abb. 1 Definition der Integrativen Onkologie.

schen Leitlinien“ gegründet (Leitung: Prof. Dr. med. Jost Langhorst), die sich zum Ziel gesetzt hat, evidenzbasierte Erfolge der Komplementärmedizin im Sinne einer Integrativen Medizin in die Behandlungsrichtlinien einzubringen. Auch gibt es inzwischen verschiedene Angebote zur komplementärmedizinischen Weiterbildung für Onkologen, nicht zuletzt an den Kliniken Essen-Mitte.

\section{Das Essener Modell}

Das erste erfolgreiche klinische Pilotvorhaben in Deutschland war der Aufbau einer stationären Abteilung für Integrative Onkologie an den Kliniken Essen-Mitte. Zuvor hatte das Team dieser Klinik mit einer ambulanten „Naturheilkundlichen Sprechstunde bei Brustkrebs“ an der Universitätsfrauenklinik Essen in den Jahren 2004-2008 positive Erfahrungen gesammelt. Daraus entwickelte sich 2010 eine Zusammenarbeit des Brustzentrums der Kliniken Essen-Mitte (PD Dr. Sherko Kümmel) mit dem Lehrstuhl für Naturheilkunde und Integrative Medizin der Universität Duisburg-Essen. Ihr folgten weitere Abteilungen: die Kliniken für Gynäkologie und Gynäkologische Onkologie (Leitung: Prof. Andreas DuBois), die Klinik für Internistische Onkologie / Hämatologie mit dem Zentrum für Palliativmedizin (Leitung: Prof. Hansjochen Wilke und Prof. Michael Stahl). Alle integrativen Therapieansätze werden auf Wirksamkeit und Sicherheit evaluiert, gleichzeitig wird die Forschung vorangetrieben.

Vorbild des Essener Modells ist das 2-Experten-Modell des renommierten Memorial Sloan Kettering Cancer Centers in New York, wo jährlich über 17000 Patienten durch die gemeinsame und koordinierte Expertise von Onkologen und Naturheilkunde-Experten versorgt werden. Dieser integrative Ansatz verspricht deutlich bes- sere Erfolge, als wenn onkologische Abteilungen lediglich einzelne komplementärmedizinische Ansätze als „Add-on“ übernehmen.

Die Nebenwirkungen einer Krebstherapie sind vielfältig. Sie reichen von akuten Beschwerden (z. B. Übelkeit) bis hin zu jahrelang anhaltenden Folgen (z.B. Fatigue). Häufig zeigen sich Hautveränderungen oder gastrointestinale Beschwerden. Antihormonelle Behandlungen von hormongetriggerten Tumoren (bei Frauen Brustkrebs, bei Männern Prostatakrebs) können zu massiven menopausalen Symptomen wie Hitzewallungen, Gelenkschmerzen oder Depression führen. Die erfolgreiche Linderung von Hitzewallungen durch Anwendung von komplementärmedizinischen Verfahren sowie die Verringerung von Gelenkschmerzen unter Therapien mit Aromatasehemmern sind wissenschaftlich gut belegt.

Etwa 60\% der Essener Patientinnen nehmen deshalb das Angebot einer integrativ-onkologischen Therapie wahr. Dazu zählt eine ausführliche Beratung über das Management von Nebenwirkungen der onkologischen Therapie durch einen naturheilkundlich ausgerichteten Arzt. Das am häufigsten eingesetzte Therapieverfahren ist die Akupunktur, deren Wirksamkeit durch viele Studien belegt ist. Sie wird während oder nach der Chemotherapie eingesetzt, während der Strahlentherapie, während der antihormonellen Therapie sowie bei Schmerzen. Jährlich finden mehr als 2000 Akupunktursitzungen statt.

\section{Basis: Mind-Body-Medizin}

Eine besonders wichtige Basis der Integrativen Onkologie ist die Mind-Body-Medizin, die bei der mentalen und psychischen Verarbeitung des Krankheitsgeschehens hilft. Dabei geht es im Kern um

\section{Wesentliche Ziele der Integrativen Onkologie sind eine} erfolgreiche Symptomkontrolle und ein verbessertes Coping. Als supportive Therapie unterstützt sie die konventionelle onkologische Behandlung, indem sie die Compliance verbessert. 
gesundheitsförderliche Bewegung, richtige Ernährung und eine Schulung zum Umgang mit Stress. Eine prospektive Studie wies bei Brustkrebspatientinnen nach, dass bereits ein moderates Sportprogramm (3 Stunden wöchentlich) in Kombination mit einem erhöhten Anteil an Obst und Gemüse (5-mal täglich) das 10-JahresMortalitätsrisiko halbiert [8]. Auch die Evidenz achtsamkeitsbasierter Therapien wie die Mindfulness-Based Stress Reduction (MBSR), Yoga und Qigong ist gut untersucht.

Im Gegensatz zur klassischen Psychoonkologie kommt die Mind-Body-Medizin (MBM) nicht erst zum Einsatz, wenn eine seelische Störung durch die Erkrankung diagnostiziert wurde. Der salutogenetische Ansatz der Integrativen Onkologie ist bemüht, die individuellen Ressourcen der Patienten so früh wie möglich zu stärken - Mind-Body-Therapien werden deshalb ab dem Moment der Diagnosestellung angeboten. Studien von Susan Lutgendorf, Psychoneuroimmunologin an der Universität von Iowa, unterstreichen die Bedeutung des Einsatzes von MBM zur Stressreduktion: Sie zeigen, dass die Signalketten der Krebsentstehung auf jeder Stufe (Entartung, Wanderung, Invasion, Ausbildung von Gefäßen zur Nährstoffversorgung, Vermehrung) beeinflussbar sind - positiv wie negativ. Die Mind-Body-Medizin wirkt über epigenetische Schalter regulierend und dämpfend auf das Krankheitsgeschehen ein [9]. Das ist auch deshalb wichtig, weil eine Depression einer Metaanalyse zufolge eine um 39\% erhöhte Mortalität nach sich zieht [10].

Studien belegen auch die antidepressive Wirkung von Sport / Bewegung [11], Achtsamkeitsmeditation [12], Yoga [13], von Gruppenunterstützung [14] sowie einer Anhebung der Omega-3-Fettsäuren in der Ernährung [15]. Im Gegensatz zur medikamentösen Behandlung der Depression besteht bei den Mind-Body-Therapien nicht die Gefahr von Cytochrom-P450-Reaktionen (Wechselwirkungen mit Chemotherapie bzw. Bestrahlung).

Mind-Body-Techniken müssen über einen längeren Zeitraum erlernt werden. In Anlehnung an Konzepte des Mind/Body Medical Institute der Harvard Medical School, USA, findet in Essen in einer

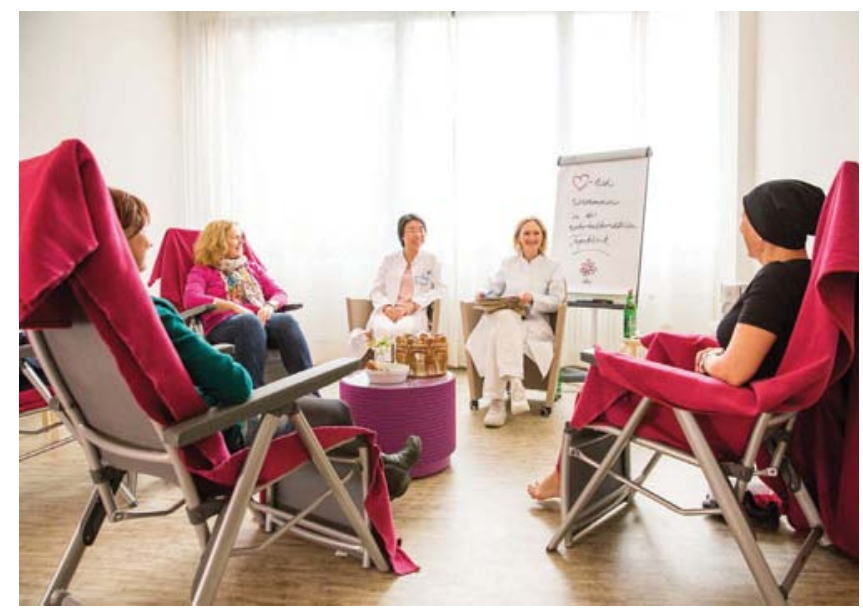

(c) Kliniken Essen-Mitte

teilstationären Tagesklinik u.a. ein 11-wöchiges Gruppenprogramm speziell für Krebspatienten statt. Vermittelt werden medizinische Informationen, Lebensstilfaktoren, Achtsamkeitsschulung, Stressbewältigung und naturheilkundliche Selbsthilfestrategien - an jeweils einem Tag in der Woche für 6 Stunden. Abb. 2 zeigt ein Beispielprogramm einer solchen Tagesklinik.

Eine eigene (offene) Gruppe bietet Chemotherapie-Patienten die Möglichkeit, sich am Vortag ihres jeweils nächsten Behandlungszyklus zu entspannen, körperlich zu stärken und seelisch motivieren zu können. Die Möglichkeit einer flexiblen Teilnahme wurde bewusst in das Konzept integriert, um die Tagesklinik an die spezifischen Bedürfnisse von Patienten während einer Chemotherapie anzupassen.

\section{Phytotherapie, Misteltherapie, Nahrungsergänzung}

Neben Akupunktur und Mind-Body-Medizin kommen in Essen auch Phytotherapie, Beratungen zu Vitaminen und Spurenelementen, Misteltherapie und Fußmassage zum Einsatz.

Abb. 2 Übersichtsplan einer onkologischen Tagesklinik an den Kliniken Essen-Mitte.

\section{Naturheilkundliche onkologische Tagesklinik Mind/ Body Medicine}

\begin{tabular}{|c|c|c|c|c|c|c|c|}
\hline \multicolumn{8}{|c|}{$\begin{array}{l}\text { Ernährungstherapie } \\
\text { Bewegungstherapie } \\
\text { Entspannung (RR) }\end{array}$} \\
\hline 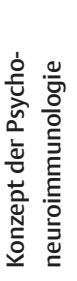 & 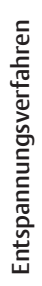 & 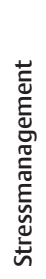 & 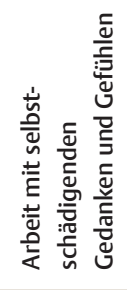 & $\begin{array}{l}\text { ত্ } \\
\text { ঠ }\end{array}$ & 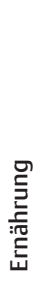 & 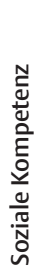 & 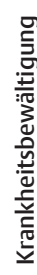 \\
\hline & & & Ilkundlich & & & & \\
\hline
\end{tabular}




\section{Die wichtigsten durch Studien belegten Erfolge der Integrativen Onkologie}

- Hilfe bei Übelkeit und Erbrechen [16-18]

- Minderung klimakterischer Beschwerden bei antihormoneller Behandlung, z. B. durch Cimicifugaextrakte $[19,20]$, Leinsamenschrot [21], Akupunktur [22, 23] oder gruppentherapeutische Intervention mit Meditationen und Atemübungen [24]

- Minderung der chronischen Müdigkeit (Fatigue) [25-27]

- Minderung von Schleimhautveränderungen und Mundtrockenheit $[28,29]$

- Minderung von Nagelveränderungen [30]

- Minderung von Durchfall oder Verstopfung

- Minderung von Hand-Fuß-Syndrom sowie Neuropathie [31, 32]

- Linderung von Schmerz

- Besserung von Schlaflosigkeit, Angst und Depression [33-40]

- Verbesserung der Lebensqualität [41]

Dass die kontinuierliche Absprache zwischen Onkologie und wissenschaftlicher Naturheilkunde ganz zentral für die Integrative
Onkologie ist, zeigen die Risiken eines unkoordinierten Einsatzes komplementärer - und erst recht alternativer - Therapien. Einige Heilkräuter, aber auch synthetische Medikamente, Grapefruitsaft, grüner Tee oder Nahrungsergänzungsmittel können die Chemooder Radiotherapie massiv beeinträchtigen, v.a. über das CYPEnzymsystem, das eine wichtige Rolle im Stoffwechsel des Darms spielt. CYP-Hemmer bedingen, dass die Wirkung onkologischer Therapien zunimmt, allerdings auch die Nebenwirkungen. CYP-Induktoren sorgen hingegen dafür, dass Substrate schneller abgebaut werden, das kann potenziell sogar ein Therapieversagen auslösen.

$\mathrm{Zu}$ beachten sind spezifische mögliche Wechselwirkungen zwischen pflanzlichen Wirkstoffen und Cytochrom-P450-Enzymen sowie P-Glykoproteinen, die Transportfunktionen erfüllen und Einfluss auf den Metabolismus haben. Cyp3A4 ist der Hauptschutzmechanismus in der Leber und verantwortlich für den sog. „first pass effect“. Diesen Hauptmetabolisierungsweg nehmen viele Medikamente, die in der onkologischen Therapie eingesetzt werden, wie z. B. der Tyrosinkinaseinhibitor Lapatinib, aber auch das natürliche Antidepressivum Johanniskraut.

Die Integrative Onkologie kann also auch wesentlich dazu beitragen, dass unerwünschte Effekte durch Selbstbehandlung ausbleiben und selbst ernannten Heilern, die von den Patienten aufgesucht werden, wenn der Onkologe sich nicht ausreichend um sie kümmern kann, das Wasser abgräbt.

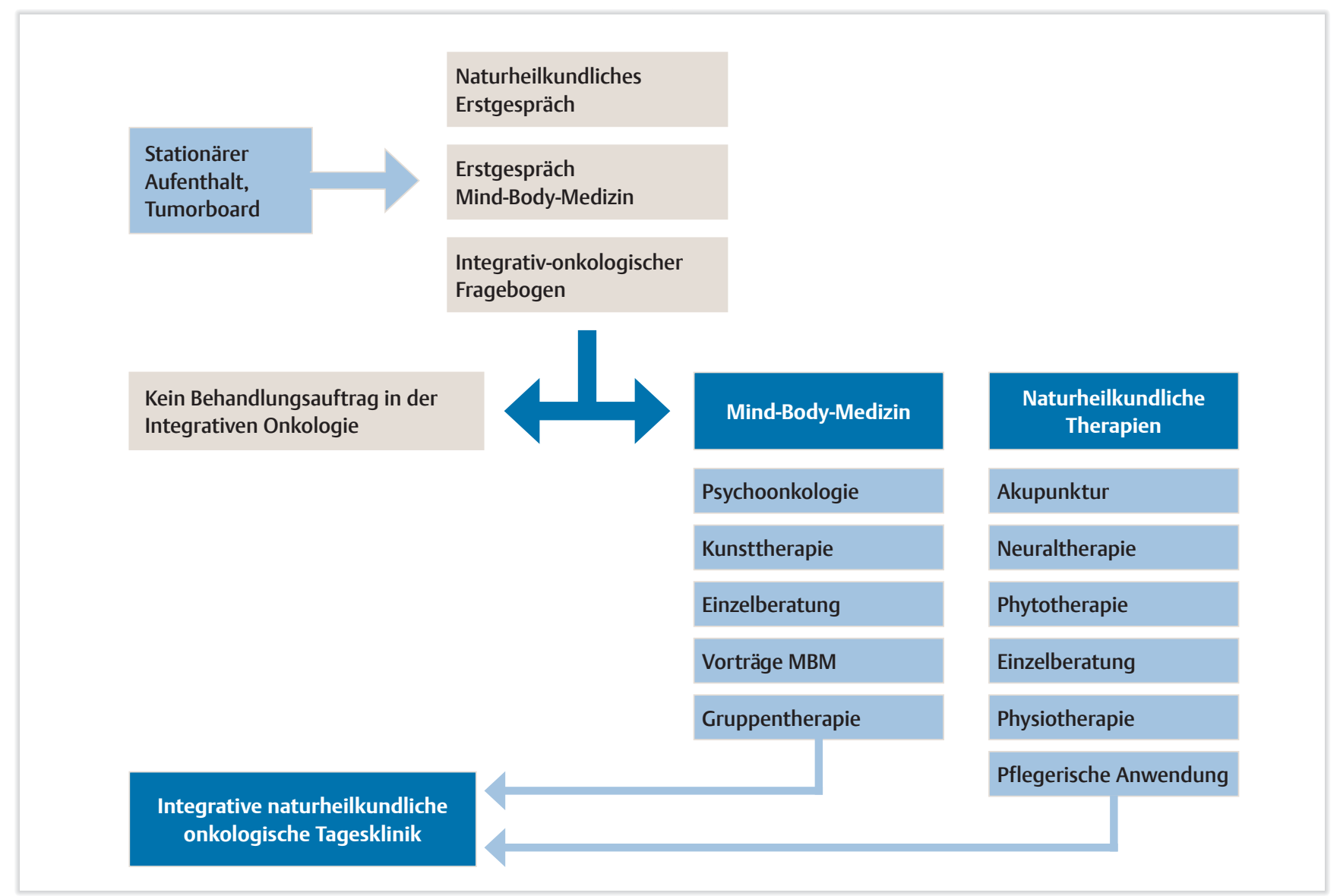

Abb. 3 Verknüpfung der komplementären und konventionellen Angebote im Rahmen der Integrativen Onkologie an den Kliniken Essen-Mitte. 


\section{Stationäre Behandlung/Fachambulanzen für Integrative Onkologie}

- Essen: Klinik für Naturheilkunde und Integrative Medizin / Brust-Zentrum der Kliniken Essen-Mitte

- Filderstadt: Filderklinik, Zentrum für Integrative Onkologie

- Hamburg: Ambulanz Chinesische Medizin, Mammazentrum am Krankenhaus Jerusalem

- Herdecke: Komplementäre Onkologie im Gemeinschaftskrankenhaus Herdecke

- Jena: Ambulanz für Naturheilkunde und Integrative Onkologie, Universitätsklinikum Jena

- Kassel/Bad Wilhelmshöhe: Habichtswaldklinik, Abteilung Onkologie

- Öschelbronn: Centrum für Integrative Medizin, Klinik Öschelbronn

- St. Gallen: Kantonsspital, Zentrum für Integrative Medizin

- Zürich: Universitätsspital, Institut für komplementäre und integrative Medizin

\section{Forschung und Ausbildung}

Die Patienten sind längst gewonnen, wenn es um das Potenzial der Integrativen Onkologie geht. Eine Hürde in der Verbreitung sind aber immer noch Vorurteile der naturwissenschaftlich orientierten „Schulmedizin“, die nur durch Aufklärung und Wissen bekämpft werden können. Ein wichtiger Baustein dafür ist ein Curriculum „Integrative Onkologie“, das auf der Basis eines von der Robert Bosch Stiftung geförderten Consensus-Gesprächs von 20 internationalen und interdisziplinären Experten erarbeitet wurde [42]. Der Workshop legte über eine ausführliche Zusammenschau der Evidenzen in diesem Bereich auch die Basis für erste komplementärmedizinische Leitlinien im Bereich Onkologie.

Als erste onkologische Fachgesellschaft hat die Arbeitsgemeinschaft Gynäkologische Onkologie (AGO) komplementärmedizinische Verfahren 2002 in ihre Leitlinien integriert und 2011 um Verfahren wie MBSR, Yoga und Qigong erweitert. Im Winter 2013 wurde nun als erste naturheilkundliche Fachgesellschaft die „Gesellschaft für Phytotherapie“ (GPT) in die Arbeitsgemeinschaft der Wissenschaftlichen Medizinischen Fachgesellschaften AWMF aufgenommen.

Eine ursächliche tumormedizinische Behandlung durch naturheilkundliche Therapien steckt noch in den Kinderschuhen, was die Nachweise ihrer Wirksamkeit angeht. Ein großes Potenzial, das z.T. bereits vom Deutschen Krebsforschungszentrum erforscht wird, haben verschiedene Pilze und Heilkräuter aus China. Studien zeigen einzelne Erfolge bei der Kombination von chinesischen Kräutern in Kombination mit einer platinbasierten Chemotherapie gegen nichtkleinzelligen Lungenkrebs [43] oder therapieunterstützend bei einer Chemotherapie gegen Darmkrebs (Noto-Ginseng) - gleichzeitig mildern andere Kräuter die Nebenwirkungen [44, 45].

Das Deutsche Cochrane Zentrum hat eine Arbeitsgruppe Biologische Krebsmedizin am Klinikum Nürnberg eingerichtet [46].

Die sachbezogene Auseinandersetzung im Rahmen der Integrativen Onkologie ist ein entscheidender Schritt in der Anerkennung 


\section{Ausbildungsmöglichkeit Mind-Body-Medizin}

Bei der jährlich stattfindenden Mind Body Medicine

Summer School können sich Ärzte und Therapeuten von

Experten über die integrativen Behandlungsansätze bei

Krebs informieren lassen: 2016 wird das die Psychologin

Susan Lutgendorf von der Universität lowa sein, deren

Schwerpunkt die Psychoneuroimmunologie des Krebses ist.

Info unter: www.mindbodymedicine.de

der Leistungen der wissenschaftlichen Naturheilkunde. Ihre Glaubhaftigkeit beruht längst nicht mehr auf historischer Überlieferung oder bestimmten Weltsichten, sondern auf objektiv überprüfbaren wissenschaftlichen Fakten im Austausch internationaler Forschung. Dennoch ist die sog. „Schulmedizin“ der Diskussion über die Integration naturheilkundlicher Therapien lange ausgewichen.

Eine weitere Hürde ist die Forschungsfinanzierung, die im Wesentlichen auf der Unterstützung durch Stiftungen beruht. Wichtig wäre eine größere Unterstützung der großen Forschungsunternehmen, da in Deutschland die Forschung bisher lediglich durch Stiftungen und private Spenden getragen wird. Weitere Forschung ist dringend erforderlich - auch weil die onkologischen Behandlungsstrategien ständig erweitert werden, was neue Beschwerdebilder an Nebenwirkungen nach sich zieht.

\section{Fazit}

Gemeinsam gegen Krebs - in der Zukunft wird die demographische Entwicklung die Zahl der Tumorerkrankten weiter ansteigen lassen. Krebs wird zunehmend zu einer chronischen Erkrankung. Die Integrative Onkologie gibt den Patienten Möglichkeiten an die Hand, selbst aktiv zu werden - ein Wunsch, der von diesen immer wieder geäußert wird. Sie können ihre Nebenwirkungen mildern, ihre Lebensqualität verbessern und vielleicht sogar ihre Heilungschancen verbessern, auch wenn dieser letzte Punkt noch nicht in Studien belegt werden konnte. In der Integrativen Onkologie werden die Patienten zu Partnern des onkologischen Arztes - heimliche Behandlungsversuche von Laien sollten genauso ein Ende haben wie das Schisma zwischen der Schulmedizin und der wissenschaftlichen Naturheilkunde.

Interessenkonflikt: Die Autoren erklären, dass keine wirtschaftlichen oder persönlichen Verbindungen bestehen.

Online zu finden unter

http://dx.doi.org/10.1055/s-0036-1581997

\footnotetext{
Literatur

$\overline{1}$ Beadle GF et al. Beliefs and practices of patients with advanced cancer: implication for communication. Br J Cancer 2004; 91: 254-257

$\overline{2}$ Sagar SM et al. Integrative oncology: a Canadian and international perspective. Curr Oncol 2008; 15 (s2): s71-s73

3 Fair W. Back to the future - the role of complementary medicine in urology 1999; 162: 411-442
}

$\overline{4}$ Deng GE, Frenkel M, Cohen L et al. Evidence based clinical practice guidelines for integrative oncology: complementary therapie and botanicals. J Soc Integr Oncol 2009; 7 (3): 85-120

5 Cassileth BR, Deng GE, Gomez JE et al. Complementary therapies and integrative oncology in lung cancer: ACCP evidence-based clinical practice guidelines (2nd edition). Chest 2007; 132 (3 Suppl): 340s-354s

$\overline{6}$ Deng GE, Rausch SM, Jones LW et al. Complementary therapies and integrative medicine in lung cancer: diagnosis and management of lung cancer, 3 rd ed: American College of Chest Physicians evidence-based clinical practice guidelines. Chest 2013; 143 (5 Suppl): e420S-436S

7 Greenlee H, Balneaves L, Carlson LE et al. Clinical practice guidelines on the use of integrative therapies as supportive care in patients treated for breast cancer. J Natl Cancer Inst Monograph 2014; 50: 346-358

8 Pierce JP, Stefanick ML, Flatt SW et al. Greater survival after breast cancer in physically active women with high vegetable-fruit intake regardless of obesity. J Clin Oncol 2007; 25: 2345-2351

9 Nagaraja AS, Armaiz-Pena GN, Lutgendorf SK et al. Why stress is BAD for cancer patients. J Clin Invest 2013; 123 (2): 558-560

$\overline{10}$ Satin JR et al. Depression as a predictor of disease progression and moritality in cancer patients: a meta-analysis. Cancer 2009; 115: 5349-5361

$\overline{11}$ Mead GE et al. Exercise for depression. Cochrane Database Syst Rec 2009; (3) CD 004366

$\overline{12}$ Chiesa A, Serretti A. Mindfulness-based stress reduction for stress management in healthy people: a review and meta-analysis. J Altern Complement Med 2009; 15: 593-600

$\overline{13}$ Pilkington $\mathrm{K}$ et al. Yoga for depression: the research evidence. J Affect Disord 2005; 89: 13-24

$\overline{14}$ Goodwin PJ et al. The effect of group-psychosocial support on survival in metastatic breast cancer. N Engl J Med 2001; 345: 1719-1726

$\overline{15}$ Sarris J et al. Adjuvant use of nuitritional and herbal medicines with antidepressants, mood stabilizers and benzodiazipenes. J Psychiatr Res 2010; 44: $32-41$

$\overline{16}$ Ezzo JM et al. Acupuncture-point stimulation for chemotherapy-inducednausea or vomiting. Cochrane Database Syst Rev. 2006 Apr 19; (2):CD002285

$\overline{17}$ Streitberger K, Ezzo J, Schneider A. Acupuncture for nausea and vomiting: an update of clinical and experimental studies. Autonomic Neuroscience 2006; 129 (1-2): 107-117

$\overline{18}$ Collins KB, Thomas DJ. Acupuncture and acupressure for the management of chemotherapy-induced nausea and vomiting. J Am Acad Pract 2004; 16 (2): 76-80

$\overline{19}$ Walji R, Boon H, Guns E et al. Black cohosh (Cimicifuga racemosa [L.] Nutt.): safety and efficacy for cancer patients. Support Care Cancer 2007; 15 (8): 913-921

$\overline{20}$ Rostock M, Fischer J, Mumm A et al. Black cohosh (Cimifuga racemosa) in tamoxifen-treated breast cancer patients with climacteric complaints - a prospective observational study (accepted). Gynecol Endocrinol 2011; 27 (10): 844-848

$\overline{21}$ Pruthi S, Thompson SL, Novotny PJ et al. Pilot evaluation of flaxseed for the management of hot flashes. J Soc Integr Oncol 2007; 5 (3): 106-112

$\overline{22}$ Frisk J, Källstrom AC, Wall N et al. Acupuncture improves health-related quality-of-life (HRQoL) and sleep in women with breast cancer and hot flushes. Support Care Cancer 2012; 20 (4): 715-724

$\overline{23}$ Liljegren A, Gunnarsson P, Landgren BM et al. Reducing vasomotor symptoms with acupuncture in breast cancer patients treated with adjuvant tamoxifen: a randomized controlled trial. Breast Cancer Res Treat 2012; 135 (3): 791-798

$\overline{24}$ Carson JW, Carson KM, Porter LS et al. Yoga of awareness program for menopausal symptoms in breast cancer survivors: results from a randomized trial. Support Care Cancer 2009; 17 (10): 1301-1309 
$\overline{25}$ Vickers AJ. Acupuncture for postchemotherapy fatigue: a phase II study. J Clin Oncol 2004; 22 (9): 1731-1735

$\overline{26}$ Baumann FT, Drosselmeyer N, Leskaroski A et al. 12-week resistance training with breast cancer patients during chemotherapy: effects on cognitive abilities. Breast Care 2011; 6: 142-143

$\overline{27}$ Demiralp M, Oflaz F, Komurcu S. Effects of relaxation training on sleep quality and fatigue in patients with breast cancer undergoing adjuvant chemotherapy. J Clin Nurs, 2009; 19: 1073-1083

$\overline{28}$ Johnstone PA, Peng YP, May BC et al. Acupuncture for pilocarpine-resistant xerostomia following radiotherapy for head and neck malignancies. Int J Radiat Oncol Biol Phys 2001; 50 (2): 353-357

$\overline{29}$ Pfister DG, Cassileth BR, Deng GE et al. Acupuncture for pain and dysfunction after neck dissection: results of a randomized controlled trial. J Clin Oncol 2010; 28 (15): 2565-2570

$\overline{30}$ Scotté F, Tourani JM, Banu E et al. Multicenter study of a frozen glove to prevent docetaxel-induced onycholysis and cutaneous toxicity of the hand. J Clin Oncol 2005; 23: 4424-4429

$\overline{31}$ Schröder S, Liepert J, Remppis A et al. Acupuncture treatment improves nerve conduction in peripheral neuropathy. Eur J Neurol 2007; 14 (3): 276-281

$\overline{32}$ Wong R, Sagar S. Acupuncture treatment for chemotherapy-induced neuropathy - a case series. Acupuncture in Medicine 2006; 24 (2): 87-91

$\overline{33}$ Wang H, Qi H, Wang BS et al. Is acupuncture beneficial in depression: a meta-analysis of 8 randomized controlled trials? J Affect Disord 2008; 111 : 125-134

$\overline{34}$ Matchim Y, Armer JM, Stewart BR. Mindfulness-based stress reduction among breast cancer survivors: a literature review and discussion. Oncology Nurs Forum 2011; 38 (2): 61-71

$\overline{35}$ Ott MJ, Norris RL, Bauer-Wu SM. Mindfulness meditation for oncology patients: a discussion and critical review. Integr Cancer Ther 2006; 5 (2): 98-108

$\overline{36}$ Shennan C, Payne S, Fenlon D. What is the evidence for the use of mindfulness-based interventions in cancer care? A review. Psychooncology 2011 20 (7): 681-697

$\overline{37}$ Musial F, Büssing A, Heusser $P$ et al. Mindfulness-based stress reduction for integrative cancer care - a summary of evidence. Forsch Komplementmed 2011;18 (4): 192-202

$\overline{38}$ Lin KY, Hu YT, Chang KJ et al. Effects of yoga on psychological health, quality of life, and physical health of patients with cancer: a meta-analysis. Evid Based Complement Alternat Med 2011; 659876

$\overline{39}$ Vadiraja HS, Raghavendra RM, Nagarthna R et al. Effects of a yoga program on cortisol rhythm and mood states in early breast cancer patients undergoing adjuvant radiotherapy: a randomized controlled trial. Integr Cancer Ther 2009; 8 (1): 37-46

$\overline{40}$ Smith KB, Pukall C. An evidence-based review of yoga as a complementary intervention for patients with cancer. Psychooncology 2009; 18: 465-475 $\overline{41}$ Kienle GS, Glockmann A, Schink M et al. Viscum album L. extracts in breast and gynaecological cancers: a systematic review of clinical and preclinical research. J Exp Clin Cancer Res 2009; 28: 79 doi: 10.1186/1756-9966-28-79.

$\overline{42}$ Dobos G, Hrsg. Integrative Onkologie am Beispiel Brustkrebs. Was kann die Naturheilkunde in der Tumormedizin leisten. München: Elsevier; 2014

$\overline{43}$ McCulloch M, See C, Shu XJ et al. Astragalus-based Chinese herbs and platinum-based chemotherapy for advanced non-small-cell lung cancer: meta-analysis of randomized trials. J ClinOncol 2006; 24 (3): 419-430

$\overline{44}$ Wang CZ, Luo X, Zhang B et al. Notoginseng enhances anti-cancer effect of 5 -fluorouracil on human colorectal cancer cells. Cancer Chemother Pharmacol 2007; 60 (1): 69-79

$\overline{45}$ Taixiang W, Munro AJ, Guanjian L. Chinese medical herbs for chemotherapy side effects in colorectal cancer patients. Cochrane Database Syst Rev 2005; Jan 25;(1).

$\overline{46}$ www.cochrane.de/de/biologische-krebstherapie

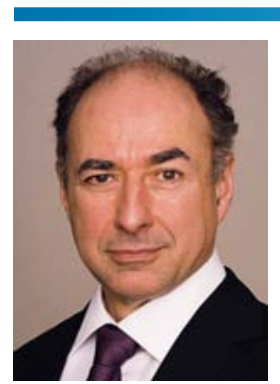

Prof. Dr. med. Gustav Dobos

Kliniken Essen-Mitte

Knappschafts-Krankenhaus

Am Deimelsberg 34a

45276 Essen

gustav.dobos@uni-duisburg-essen.de

Gustav Dobos ist Internist, Nephrologe und Intensivmediziner mit den Zusatzbezeichnungen Akupunktur, Naturheilkunde, Physikalische Therapie, Chirotherapie. Seit 1999 leitet er die Klinik für Naturheilkunde und Integrative Medizin im Knappschafts-Krankenhaus der Kliniken Essen-Mitte. Im September 2004 erhielt er den Ruf auf den Alfried Krupp von Bohlen und Halbach-Stiftungslehrstuhl für Naturheilkunde der Universität Duisburg-Essen. Vorsitzender der Deutschen Gesellschaft für Naturheilkunde (DGFN). 